20

\title{
Лазерный корреляционный спектрометр для оценки размеров и динамики изменения размеров структур в биологических жидкостях
}

\author{
(C) Е.Н. Величко ${ }^{1}$, Э.К. Непомнящая ${ }^{1}$, А.В. Соколов ${ }^{2}$, Т.Ю. Кудряшова ${ }^{1}$ \\ ${ }^{1}$ Санкт-Петербургский политехнический университет Петра Великого, \\ 195251 Санкт-Петербург, Россия \\ ${ }^{2}$ АО „Концерн „ЦНИИ „Электроприбор“, \\ 197046 Санкт-Петербург, Россия \\ e-mail: elina.nep@gmail.com
}

Поступила в редакцию 10.12.2019 г.

В окончательной редакции 03.02.2020 г.

Принята к публикации 28.02.2020 г.

Для оценки размеров наноструктур в биологических жидкостях и исследования динамики их изменения в работе предложен модифицированный метод лазерной корреляционной спектроскопии. Описаны схема аппаратно-программного комплекса и алгоритм метода, позволяющий добиться высокой точности определения размеров наночастиц, а также исследовать процесс изменения размеров наночастиц в динамике.

Предложенный аппаратно-программный комплекс позволил провести исследования динамики образования агрегатов в сыворотке крови человека в процессе иммунного ответа. Полученные результаты свидетельствуют о наличии процессов быстрой агрегации белков в результате активации иммунного ответа, кроме того размер образующихся агрегатов зависит от состояния иммунной системы и наличия заболеваний.

Ключевые слова: лазерная корреляционная спектроскопия, динамическое рассеяние света, размеры частиц, наночастицы, биологическая жидкость, молекулярная агрегация.

DOI: $10.21883 /$ OS.2020.07.49567.63-20

\section{Введение}

В настоящее время основные диагностические данные о состоянии организма человека получают с помощью исследования крови и других биологических жидкостей [1]. При этом немаловажно не только определение общих концентраций веществ в крови, но и анализ функциональности тех или иных биологических систем, в том числе молекулярных комплексов. Прямых методов для подобного анализа в современной диагностической медицине не внедрено.

В данной работе в качестве диагностического параметра для определения функциональности молекулярных комплексов предлагается использование размера частиц в сыворотке крови. Известно, что различные типы структур в крови отличаются не только биохимическими свойствами, но и размерами [2]. При этом ряд процессов, происходящих в организме человека, сопровождается агрегацией молекулярных структур и, соответственно, изменением их размеров $[3,4]$.

Таким образом, анализируя размер структур в жидкости можно определить ее состав и проанализировать динамику происходящих в ней биологических процессов. Существующие в настоящее время методы для определения размеров структур имеют ряд недостатков, ограничивающих их применение в медицинской диагностике. В табл. 1 приведен краткий обзор существующих методов оценки размеров наночастиц.
Как уже было отмечено, важна возможность оценки изменения размеров структур в биологических жидкостях в динамике. Из таблицы видно, что большинство существующих методов не позволяют проводить исследования частиц с размерами менее $100 \mathrm{~nm}$ в динамике. Перспективным представляется развитие методов лазерной корреляционной спектроскопии [5]. Ограниченное применение лазерной корреляционной спектроскопии в медицине в настоящее время связано с недостаточной точностью и совершенством существующих приборов и алгоритмов обработки данных. В данной работе предлагается схема аппаратно-программного комплекса (АПК) и алгоритм проведения измерений, разработанные для реализации возможности оценки динамики агрегации молекулярных структур в биологических жидкостях [6].

\section{Схема и алгоритм проведения измерений}

Метод лазерной корреляционной спектроскопии основан на измерении временной зависимости интенсивности рассеянного излучения на частицах, совершающих броуновское движение в жидкости [7]. Спектр такого рассеяния будет иметь допплеровское уширение $\Delta f$, зависящее от коэффициента диффузии частиц, который в свою очередь зависит от их размеров согласно формуле

$$
\Delta f=q^{2} D_{T} / 2 \pi=q^{2} k_{b} T / 6 \pi^{2} \eta d .
$$


Таблица 1. Основные методы оценки размеров наночастиц

\begin{tabular}{l|c|l}
\hline \multicolumn{1}{c|}{ Методы анализа } & $\begin{array}{c}\text { Определяемые размеры } \\
\text { частиц, nm }\end{array}$ & Недостатки \\
\hline Сканирующая электронная микроскопия & $5-5000$ & Невозможность оценки динамики \\
\hline Просвечивающая электронная микроскопия & $5-5000$ & Невозможность оценки динамики \\
\hline Рентгенография & $0.5-1000$ & $\begin{array}{l}\text { Сложность подготовки проб, } \\
\text { невозможность оценки динамики }\end{array}$ \\
\hline Седиментационный анализ & $100-10000$ & $\begin{array}{l}\text { Низкая точность при исследовании } \\
\text { молекулярных комплексов }\end{array}$ \\
\hline Оптическая микроскопия & $1000-100000$ & Неподходящий диапазон размеров \\
\hline Статическое светорассеяние & $500-8000$ & Неподходящий диапазон размеров \\
\hline Лазерная корреляционная спектроскопия & $0.5-2000$ & $\begin{array}{l}\text { Несовершенство существующих } \\
\text { приборов и алгоритмов }\end{array}$
\end{tabular}

Здесь $\Delta f$ - допплеровское уширение, $D_{T}-$ коэффициент диффузии, $\eta$ - вязкость жидкости, $k_{b}-$ постоянная Больцмана, $T$ - температура, $d-$ гидродинамический диаметр частиц, $q-$ модуль вектора рассеяния

$$
q=\frac{4 \pi n_{0}}{\lambda_{0}} \sin \left(\frac{\theta}{2}\right),
$$

где $n_{0}$ - показатель преломления среды, $\lambda_{0}-$ длина волны излучения источника, $\theta-$ угол регистрации рассеяния.

В данной работе регистрация рассеянного излучения производится с использованием аппаратного комплекса, схема которого приведена на рис. 1. Принципы построения схемы и выбора элементов обсуждались нами в предыдущих работах $[8,9]$.

Для создания когерентного монохроматического излучения в работе используется полупроводниковый лазерный модуль с длиной волны $650 \mathrm{~nm}$ [10]. Для стабилизации флуктуаций интенсивности излучения питание модуля осуществляется с использованием аккумуляторной батареи, не подключенной к сети. Контроль мощности излучения в работе осуществляется перед проведением измерений с использованием измерителя мощности [11].

Задание линейной поляризации излучения происходит с использованием поляризационного фильтра, а фокусировка в объеме исследуемого раствора осуществляется системой линз. Исследуемый раствор помещается в ячейку из кварцевого стекла, прозрачного в видимом диапазоне длин волн. Рассеянное излучение детектируется при помощи фотоэлектронного умножителя, сбор и передача рассеянного излучения на входную апертуру умножителя осуществляется с использованием системы линз и оптоволоконного световода $[12,13]$.

Затем сигнал оцифровывается и передается на систему обработки $[14,15]$ и анализа данных, в роли которой выступает компьютер с установленной программой записи и обработки данных [16].

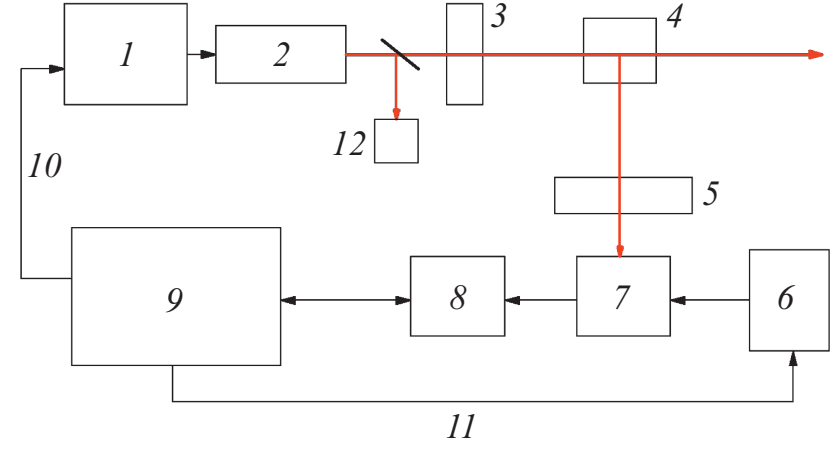

Рис. 1. Блок-схема лазерного корреляционного спектрометра. 1 - стабилизированный источник тока для питания лазерного модуля, 2 - лазерный модуль с выбранной длиной волны излучения, 3 - система фокусировки, 4 - ячейка с исследуемым образцом, 5 - система сбора излучения, 6 - стабилизированный источник питания с регулировкой усиления приемника 7 - фотоприемник, 8 - модуль оцифровки данных, $9-$ система обработки и анализа данных, 10 - линия обратной связи для регулировки мощности излучения, 11 - линия обратной связи для регулировки усиления сигнала, 12 контроль мощности зондирующего излучения.

Измерения для вычисления размеров исследуемых структур проводятся согласно приведенному ниже протоколу:

1) приготовить исследуемый раствор;

2) разместить образец в измерительной ячейке и стабилизировать тепловое равновесие между образцом и измерительной ячейкой. Колебания равновесной температуры должны быть в пределах $\pm 1^{\circ} \mathrm{C}$. Необходимость поддержания постоянной температуры и контроля ее значения обусловлена температурной зависимостью вязкости раствора, входящей в расчетную формулу для размера; 
3) подключить прибор к компьютеру и запустить программу для записи и обработки данных рассеяния света;

4) ввести в компьютерную программу данные, необходимые для проведения измерений: длительность измерения, температуру, показатель преломления и коэффициент динамической вязкости испытуемого образца, длину волны лазерного излучения и значение угла рассеяния, если эти параметры выбираются, наименование эксперимента;

5) запустить калибровочное измерение, в рамках которого на ячейку с исследуемым образцом подается короткий световой импульс длительностью $10 \mathrm{~ms}$ и происходит настройка мощности лазерного излучения [17] исходя из регистрируемой интенсивности рассеяния, определяется уровень темнового тока фотоприемника;

6) запустить запись динамического сигнала рассеяния лазерного излучения на исследуемом образце;

7) обработать полученные данные (вычислить среднее значение размера, стандартное отклонение и распределение частиц по значениям размера). При исследованиях динамических параметров требуется задать временной шаг, с которым будут вычисляться размеры.

Для обработки данных в работе используется специализированный алгоритм численного решения обратной некорректной задачи, возникающий при анализе динамического рассеяния в лазерной корреляционной спектроскопии. В основе данного алгоритма лежит модифицированный метод регуляризации Тихонова, подробное описание алгоритма можно найти в предыдущих работах $[16,18]$. Алгоритм позволяет вычислять средние размеры частиц в растворах, содержащих частицы различных размеров.

\section{Апробация аппаратно-программного комплекса}

Апробация предложенного АПК в работе производилась на модельных растворах стеклянных микросфер с заранее известными размерами. Концентрация частиц в растворах составляла $0.05 \mathrm{~g} / 1$. Результаты вычисления и сравнение их с результатами, полученными при помощи других методов, представлены в табл. 2 .

Погрешность определения размеров, представленная в таблице, вычислялась в относительных величинах исходя из измеренного значения СКО и доверительной вероятности $95 \%$.

Погрешность измерения размеров частиц в полидисперсных растворах оценивалась аналогичным образом для смеси стеклянных микросфер с диаметрами 60, 90 и $150 \mathrm{~nm}$. В табл. 3 представлены результаты проведенного анализа.

Полученные результаты позволяют оценить погрешность определения размеров частиц в моно- и полидисперсных растворах. Помимо модельных растворов в данной работе проводилось исследование размеров
Таблица 2. Расчет размеров наночастиц различных размеров и оценка погрешности

\begin{tabular}{l|r|r|r|r|r|r}
\hline Номинальный размер, $\mathrm{nm}$ & 20 & 60 & 90 & 150 & 320 & 540 \\
\hline Рассчитанный размер, $\mathrm{nm}$ & 20.6 & 61.5 & 92.3 & 147.9 & 325.3 & 547.1 \\
\hline Погрешность, \% & 3.2 & 3.2 & 3.7 & 3.9 & 3.7 & 6.1
\end{tabular}

Таблица 3. Расчет размеров наночастиц в полидисперсной смеси и оценка погрешности

\begin{tabular}{l|r|r|r}
\hline Номинальный размер, nm & 60 & 90 & \multicolumn{1}{c}{150} \\
\hline Рассчитанный размер, nm & 60.6 & 92.3 & 147.9 \\
\hline Погрешность, \% & 5.5 & 5.9 & 9.9
\end{tabular}

частиц в растворе белка альбумина. Данный белок составляет до $60 \%$ всех белков сыворотки крови и несет исключительно важную функцию для организма [19-21]. Результаты определения размеров структур в водном растворе альбумина с концентрацией $1 \mathrm{~g} / 1$ представлены на рис. 2. Здесь $d-$ диаметр частиц в растворе, $N-$ относительная концентрация частиц.

Согласно литературным данным, средний размер белка альбумина составляет порядка $6 \mathrm{~nm}$, что согласуется с результатами нашего исследования. При этом следует отметить, что в исследуемых расстворах также обнаружено присутствие более крупных структур с размерами порядка 70-100 nm, которые можно ассоциировать с агрегатами белков. Существуют свидетельства, доказывающие наличие тенденции белка альбумина в неравновесных условиях образовывать агрегаты различной формы и размеров [22]. Аналогичные исследования проводились

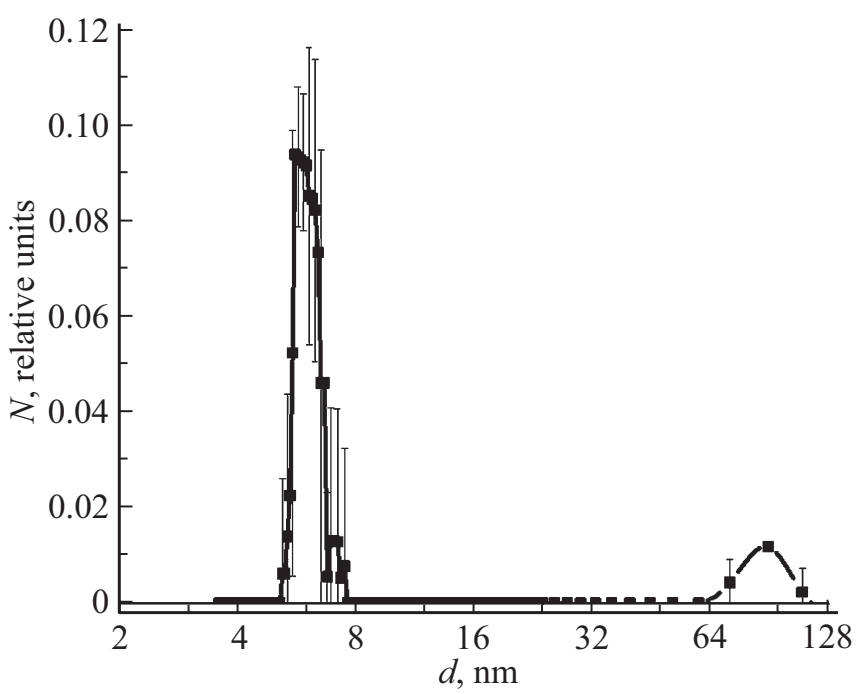

Рис. 2. Результат измерения диаметра молекулы альбумина в воде на разработанном АПК. 
другими авторами и результаты их работ согласуются с полученными нами данными [19-22].

\section{Исследование динамики агрегации}

Наибольший интерес с точки зрения развития научных исследований представляет возможность исследования динамики изменения размеров структур в биологических жидкостях в процессе их агрегации. Подобные процессы регулярно наблюдаются в организме человека в результате протекания различных реакций. Одним из примеров является агрегация белков сыворотки крови, вызванная активацией иммунного ответа организма и образованием иммунных комплексов [23]. Данные комплексы состоят из большого числа единичных белков и предназначены для идентификации чужеродных патогенов [24]. Длительность реакции активации иммунной системы, образованной системой белков комплемента [25], составляет порядка $30 \mathrm{~s}$, что создает значительные трудности при исследовании данного процесса биохимическими методами.

При помощи описанного в данной работе АПК и алгоритма проведения измерений был проведен анализ динамики активации иммунной системы в сыворотке крови здоровых доноров. Исследование проводилось до и в течение $210 \mathrm{~s}$ после активации иммунной системы при помощи антигена, в роли которого выступала вакцина „Гриппол“. В исследовании использовалась кровь 10 условно-здоровых доноров и 8 доноров с патологиями иммунного ответа.

Сыворотку, используемую для исследований, получали из цельной крови доноров в день забора материала. Для запуска процесса естественного свертывания образцы крови в стерильных пробирках $3 \mathrm{~h}$ выдерживались в термостате при температуре $37^{\circ} \mathrm{C}$, а затем для лучшего отделения сыворотки центрифугировались в течение $3 \mathrm{~min}$.

На рис. 3 представлены результаты исследования активации иммунитета в сыворотках крови здоровых доноров. На данном графике $d-$ диаметр частиц, $t-$ время, прошедшее после активации иммунной системы. В момент $t=0$ исследуются размеры структур в сыворотке до активации иммунной системы. Сразу после этого в сыворотку добавляется антиген и регистрируется начало реакции агрегации. Наблюдается постепенное увеличение размеров структур от 8 до $40 \mathrm{~nm}$ в процессе протекания иммунной реакции. Начиная примерно со 175-й s после добавления в сыворотку антигена динамических процессов не наблюдается, и можно заключить, что процесс сборки иммунных комплексов на антигене завершился.

На рис. 4 представлены усредненные результаты исследования активации иммунитета в сыворотках крови 8 доноров с нарушениями иммунного ответа, спровоцированными различными заболеваниями. В момент $t=20 \mathrm{~s}$ и далее наблюдается увеличение размеров

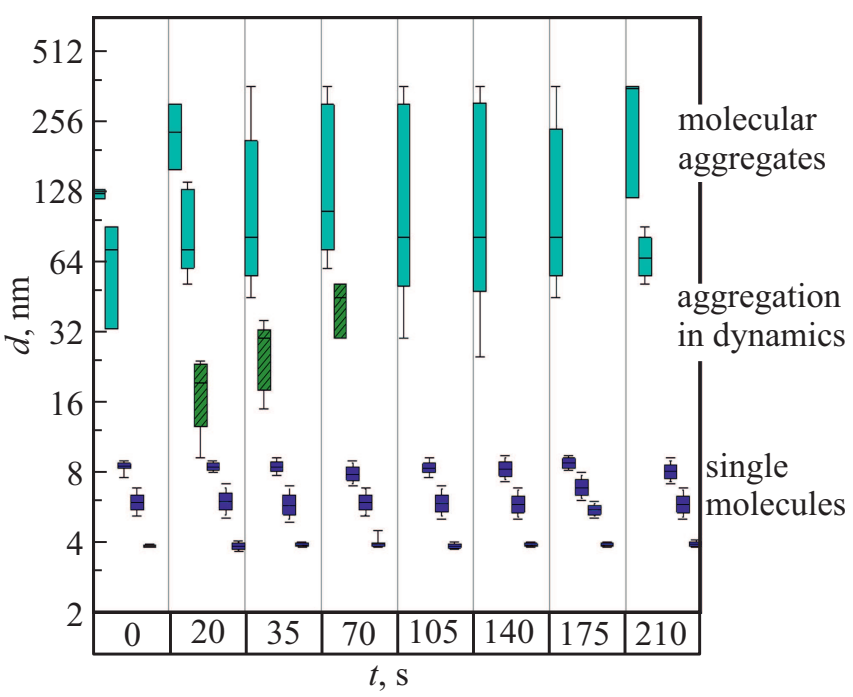

Рис. 3. Размеры структур в сыворотке крови здоровых доноров до $(0 \mathrm{~s})$ и после $(20-210 \mathrm{~s})$ добавления в сыворотку крови антигена.

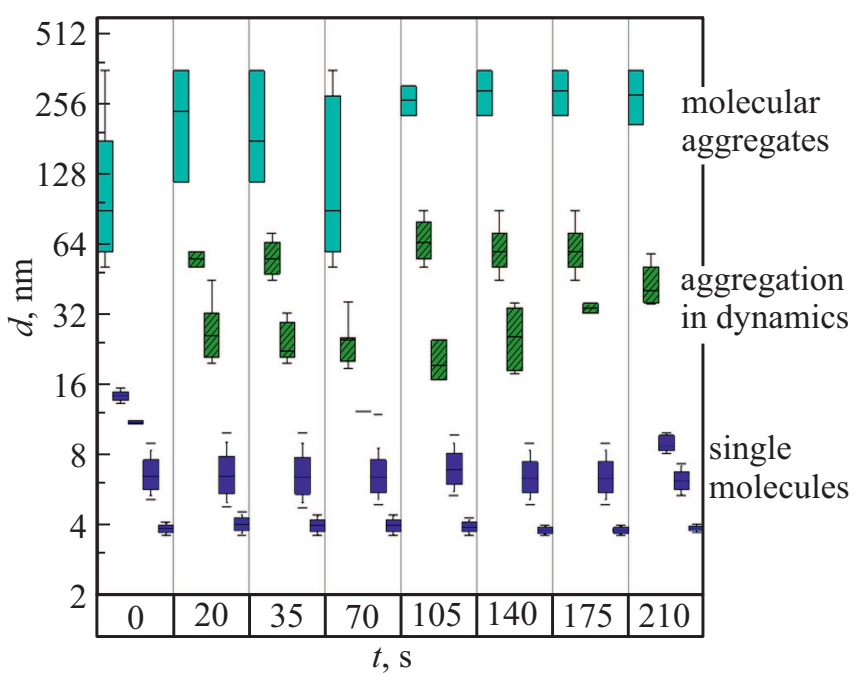

Рис. 4. Размеры структур в сыворотке крови доноров с нарушениями иммунного ответа до $(0 \mathrm{~s})$ и после $(20-210 \mathrm{~s})$ добавления в сыворотку крови антигена.

структур относительно момента времени $t=0$. Однако общая динамика активации иммунной системы отличается по сравнению с аналогичной картиной, полученной для здоровых доноров.

Наблюдается иной характер активации иммунитета и образования иммунных комплексов, нежели при исследовании сыворотки крови здоровых доноров. При нарушениях иммунного ответа видно, что в исследуемой сыворотке крови не образуется крупных структур с размерами $80-100 \mathrm{~nm}$, как в случае наблюдения иммунной реакции у здоровых доноров. После добавления в исследуемую сыворотку антигена в размерном распределении формируются структуры с размерами $18-60 \mathrm{~nm}$, постепенно увеличивающие свои размеры в ходе протекания 
иммунной реакции. Отличающаяся динамика протекания реакции по сравнению со здоровыми донорами может быть ассоциирована с нарушениями иммунного ответа.

Различия в распределении белков по размерам при активации иммунной системы с использованием антигена позволяют сделать выводы о наиболее важных свойствах биологических макромолекул, а именно об их способности образовывать высокоупорядоченные структуры и комплексы в соответствии с их конечным функциональным назначением. Исследования, проведенные с помощью разработанного аппаратно-программного комплекса, позволили наблюдать в динамике за реакцией образования молекулярных комплексов в сыворотке крови, а также обнаружить различия в протекающих иммунных реакциях у здоровых доноров и доноров с нарушениями работы иммунной системы. Такая информация поможет определить функциональные возможности иммунной системы пациента и провести диагностические тесты на наличие определенных иммунных заболеваний.

\section{Выводы}

В данной работе предложены структура и алгоритм работы аппаратно-программного комплекса для оценки размеров структур в биологических жидкостях. Проведенные расчеты элементов схемы аппаратнопрограммного комплекса и анализ требований, предъявляемых к ним, позволили достичь высокого SNR и реализовать аппаратную часть разрабатываемого комплекса в малогабаритном корпусе.

Апробация разработанного аппаратно-программного комплекса проводилась при исследовании суспензий стеклянных микросфер с известными размерами и растворов белка альбумина. Погрешность определения размеров структур при исследовании полидисперсных растворов с частицами, размеры которых не превышают $500 \mathrm{~nm}$, составила менее $10 \%$.

В работе демонстрируются возможности применения разработанного АПК для исследования динамики изменения размеров структур в биологических жидкостях, в частности исследуется динамика активации иммунной системы в сыворотке крови здоровых доноров и доноров с нарушением работы иммунной системы. Активация иммунитета проводилась путем внесения в сыворотку крови антигенов, в роли которых выступала вакцина гриппа. Реакция активации иммунной системы наблюдалась в течение 4 мин после ее инициации. Результаты показывают, что разработанный аппаратно-программный комплекс позволяет наблюдать процесс активации иммунной системы и формирование иммунных комплексов в сыворотке крови.

Применение метода лазерной корреляционной спектроскопии в медицине для исследования биологических жидкостей позволяет получить уникальные диагностические сведения о состоянии организма, а именно, измерить размеры структур и динамику их изменения в биологических жидкостях и сделать выводы о функциональных особенностях молекул, в том числе белков организма человека.

\section{Благодарности}

Авторы благодорят Т.А. Богомаз за всестороннюю помощь и ценные научные консультации.

\section{Соблюдение этических норм}

Все процедуры, выполненные в исследовании с участием людей, соответствуют этическим стандартами институционального и/или национального комитета по исследовательской этике и Хельсинкской декларации 1964 г. и ее последующим изменениям или сопоставимым нормам этики.

\section{Конфликт интересов}

Авторы заявляют, что у них нет конфликта интересов.

\section{Список литературы}

[1] Zapryanova D., Mircheva T., Denev S.A. // Revue Med Vet. 2013. V. 164. P. $150-155$.

[2] Petrova G.P. et al. // Laser Phys. 2009. V. 19. N 6. P. 1303 1307.

[3] Kirichenko M.N. et al. // XIII International Conference on Atomic and Molecular Pulsed Lasers. International Society for Optics and Photonics. 2018. V. 10614. P. 106142C.

[4] Савченко E.A., Величко E.Н., Аксенов E.T. // Учен. зап. Казан. ун-та. Сер. Физ.-матем. науки. 2018. Т. 160 / Кн. 1. C. $108-115$.

[5] Lawrie A.S. et al. // Vox Sang. 2009. V. 96. N 3. P. 206-212.

[6] Chaikov L. et al. // J. Biomed. Optics. 2015. V. 20. N. 5. P. 057003.

[7] Stetefeld J., McKenna S.A., Patel T.R. // Biophysical Rev. 2016. V. 8. N. 4. P. 409-427.

[8] Nepomnyashchaya E., Velichko E., Kotov O. // 2019 IEEE International Conference on Electrical Engineering and Photonics (EExPolytech). IEEE, 2019. P. 321-324.

[9] Nepomnyashchaya E., Aksenov E., Velichko E. // 2017 Progress in Electromagnetics Research Symposium-Spring (PIERS). IEEE. 2017. P. 3556-3562.

[10] Привалов В.Е., Шеманин В.Г. // Опт. и спектр. 1997. Т. 82. № 4. C. 700-702; Privalov V.E., Shemanin V.G. // Opt. and Spectrosc. 1997. V. 82. N 4. P. 650-652.

[11] Ivanov S.I., Lavrov A.P. // Int. Symp. Consum. Technol. ISCT. 2018. P. 51-53.

[12] Kotov O., Chapalo I., Petrov A. // IEEE Int. Conf. Electr. Eng. Photonics, EExPolytech. 2018. P. 257-620.

[13] Kotov O.I., Bisyarin M.A., Chapalo I.E., Petrov A.V. // J. Opt. Soc. Am. B: Opt. Phys. 2018. V. 35. P. 1990-1999.

[14] Liokumovich L., Muravyov K., Skliarov P., Ushakov N. // Appl. Opt. 2018. V. 57. P. 7127-7134.

[15] Ivanov S.I., Liokumovich L.B., Medvedev A.V. // Lect. Notes Comput. Sci. (including Subser. Lect. Notes Artif. Intell. Lect. Notes Bioinformatics). 2018. V. 11118 LNCS. P. 666-674. 
[16] Nepomnyashchaya E.K. // J. Phys.: Conference Ser. IOP Publishing. 2019. V. 1236. N. 1. P. 012041.

[17] Привалов В.Е., Шеманин В.Г. // Опт. и спектр. 1997. Т. 82. № 5. C. 873-875; Privalov V.E., Shemanin V.G. // Opt. Spectrosc. 1997. V. 82. N 5. P. 809-811.

[18] Nepomnyashchaya E., Antonova E. // 2018 IEEE International Conference on Electrical Engineering and Photonics (EExPolytech). IEEE, 2018. P. 136-140.

[19] Rosenoer V.M., Oratz M., Rothschild M.A. Albumin: Structure, Function and Uses. Elsevier, 2014.

[20] Vonti A.O., Ilinskii A.V., Kapralova V.M., Shadrin E.B. // Tech. Phys. 2018. V. 63. P. 908-915.

[21] Sleep D. // Expert Opinion on Drug Delivery. 2015. V. 12. N 5. P. 793-812.

[22] Баранов А.Н., Власова И.М., Салецкий А.М. // Журн. прикл. спектроск. 2004. Т. 71. № 2. С. 204-207; Baranov A.N., Vlasova I.M., Saletskii A.M. // J. Appl. Spectr. 2004. V. 71. N 2. P. 222-226.

[23] Noris M., Remuzzi G. // Seminars in Nephrology. WB Saunders. 2013. V. 33. N 6. P. 479-492.

[24] Chester K.A., Begent R.H. // Clinical and Experimental Immunology. 1984. V. 58. N 3. P. 685.

[25] Esmail H., Lai R.P., Lesosky M., Wilkinson K.A., Graham C.M., Horswell S., Wilkinson R.J. // Proceedings of the National Academy of Sciences. 2018. V. 115. N 5. P. E964-E973. 